\title{
Types, Genes, and Genotypes: the heritable basis of Galton's law.
}

\author{
Wilhelm Johannsen*
}

maximilian@alumni.reed.edu

October 9,2020

\begin{abstract}
A translation of the eighth chapter (or lecture, Achte Vorlesung) from Elemente der Exakten Erblichkeitslehre, by Wilhelm Johannsen. Published originally by Gustav Fisher (Jena) in 1909. This is the first known publication using the words "gene", "genotype", and "phenotype".

In this chapter, Johannsen first discusses Galton's principles of selection and regression. He then uses this as a point of entry into discussing the factors of inheritance, discusses deficiencies of the traditional term "Typus" or "Type" regarding some supposedly-fixed variety of organism. He notes the related problems with such then-common terms as "Anlagen" and "Pangene". He eventually settles on the term "Gene" (Gen) to describe the inherited factors, and the terms phenotype and genotype to (respectively) describe the visible form and invisible inherited Mendelian factors of organisms. He discusses the biological interpretation of these terms in different contexts, including that of pure line breeding.
\end{abstract}

\section{TRANSLATOR'S NOTE}

Throughout, I have included footnotes where appropriate; these are in italic and prefixed with MOP to distinguish them from Johannsen's notes. I have also introduced section headings where I judged appropriate; all titles are my creation.

\section{Notable Revisions to Johannsen's Text in Later Editions.}

This text was substantially revised in later editions, e.g. 1926. For information about what changed, I direct the reader to Churchill's 1974 paper 'William Johannsen and the Genotype Concept', and the slightly later textual analysis by Wanscher in 1975, 'An analysis of Wilhelm Johannsen?s genetical term "genotype" 1909-26".

\section{Contents:}

*translation by Maximilian Oliver Press 2020
- Galton's law on selection.

- The type, in Quetelet's sense.

- Will the type be shifted by selection?

- Phenotypes, genes (pangenes), and genotypical differences.

- Homozygotes and heterozygotes.

\section{GALTON'S LAW ON SELECTION}

A very widespread view is that, for each species or race, the omnipresent individual deviations from "type" (the average kind, with regard to some character) can be used as a starting point for shifting the type, through application of appropriate selection.

If this view were correct, then one would have to consider selection an important requirement for the appearance of individuals varying more and more from the original type. This means that one must see selection as the cause 
of variability in the direction of selection.

This proceeds from the rule, which we have already found, of commonplace variations (or variants) from type. If we use as an arbitrary example a dataset with mean $M=50$ and standard deviation $\sigma=211$ and assume that this data follow the binomial distribution, then we have a means (using the table on page $65^{2}$ to reconstruct the data themselves. If we have observed 1,000 individuals in total, with no variations outside the range of $M \pm 3.5 \sigma$, that means that all individuals lie between 43 and 57 units. We would for example find 16.5 (approximately 17) individuals lying between $+2 \sigma$ and $+2.5 \sigma$, e.g. between 54 and 55 units ${ }^{3}$ The mean of these 17 positively deviating individuals may be calculated as 54.5 . Let us say that we mark these individuals alone for further breeding and obtain from them 1,000 offspring (which may be easily achieved for many animals and plants), and that Galton's law of heritability is valid, with $1 / 3$ as the heritability relation. Then, in the resulting offspring generation the mean will be $50+\frac{1}{3} * 4.5=51.5$ units. The standard deviation is still $\sigma=2$, corresponding to a very slightly reduced coefficient of variation, meaning that if all 1,000 individuals lie within the range of $M \pm 3.5 \sigma$, they will all be between 44.5 and 58.5 units.

For these offspring obtained by parental selection, we would find individuals between 57 and 58.5 units that are larger than any in the parental generation, while the parental generation will contain individuals between 43 and 44.5 units. The count of individuals in this range in the offspring will be something like 3 per 10,000 according to the table on page $65^{4}$. so by no means an infinitesimally small number.

Perhaps Galton's law is valid, and variabil-

\footnotetext{
${ }^{1}$ The example comes from the flounders mentioned on page 90 of the original text.

${ }^{2} \mathrm{MOP}$ note: in the original text.

${ }^{3}$ For 10,000 individuals, $4,938-4,773=165$; see the table on page 65 of the original text.

${ }^{4}$ MOP note: In my copy of the 1909 edition, Johannsen writes 3 per 1,000, which appears to be an error when I consult the table in question and the data at hand. Possibly this is corrected in later editions.
}

ity will not actually decrease through selection (though there is no reason why this would be the case). Then selection must necessarily (we could say, "with logical necessity") lead to variations that would not otherwise appear, if it can effect any shift of the type at all. Thus, without a shift of type in the pertinent direction, the probability of these new variations will be infinitely small according to the distributions we have discussed. In other words, selection would give rise to novelties.

In this argument it is assumed that the life history of the organism in question remains the same from generation to generation. Then the comparison must be ceased, and special corrections would be introduced. However, these reservations do not meaningfully alter the facts of the matter.

Therefore, selection in a particular directionand specifically selection for many generations further than we treat here-would lead to transgression of the original limits of variation, even with a relatively small number of breeding individuals. Without selection, an exceptionally large number of individuals (mass cultures on a massive scale) would be needed to reach beyond these limits, as the variations in question are expressed with very low frequency in the population. Thereby selection has, practically spoken, had the effect of new creation! It has realized something that has never existed before.

This conception of the action of selection is very generally held, and is formally completely justified as a consequence of Galton's law. However, this law is merely statistical and not biological, as we shall soon see.

\section{Quetelet's TYPE}

We have just now been using a word whose exact meaning we must consider, the word "type" 5 . This word is generally used in quite

\footnotetext{
${ }^{5}$ MOP note: "Typus". This word is used by many early naturalists, notably De Vries. The earliest usage that I am aware
} 
different fashions, and unfortunately, often confusion ensues. What the practical breeder means with "type" in different cases is not always totally clear, given its popularity as a buzzword. We will not pursue the usage among breeders further, but rather merely note that "type" is used very vaguely in practical work. However, this word is also used in very many different ways in scientific proceedings.

Rather, let us say that the word always means only a single kind of an organism, or more exactly expressed, the measurements from a single kind (if we only consider quantitative differences). One may absolutely not connect the term "type" with any idea of a defined relationship by descent. One would thus lose oneself in a labyrinth of ambiguity. "Type" gives only the kind and has nothing to do with the idea of "kinship" in its genealogical sense.

A specific "type" may arise in different ways, and full siblings may belong to different "types", as we will see later. Some researchers describe natural phenomena, some work with collected statistical data in a mathematical fashion (essentially, workers who observe variability), and some seek to penetrate genetical questions with experiments. All may be called researchers of heredity, and each may fancy a different definition of "type". We must therefore examine what each group means by "type", whether descriptive and mathematicalstatistical workers or genetical-experimental workers. These differences turn out to be important.

We begin with that type definition that is particularly favored by researchers who work with the variability of existing stocks or populations. Here we can say that one understands "type" to mean that measure of a kind (or that intensity of a character) for which a set of individuals belonging to some stock vary. Specifically, we mean the mean value or the center of variation for this purely numerical character. Such a type is like the expression, "unity in diversity". For example, the type of the runner bean length mentioned on page 13 will be its mean, $24.36 \mathrm{~mm}$, and the type of the rays of

of is Goethe, in the late 18th century. flounders on page 11 will be about 54 . The average error of these values gives the confidence with which this type may be determined.

Quetelet puts great stock in this meaning of type. For these researchers it is the case that the population of a nation can be grouped according to the binomial distribution for body length and many other measurements, and this is the same thing as saying that such populations each have some single type according to the characters examined. In such cases, the type will be expressed through the mean value. The type of some character of a given population or race is then, after Quetelet, that measure whose deviation is equal to zero.

This applies particularly if the variations are distributed symmetrically around the mean, as is the case for the binomial distribution. Only then may one say that the variations from the rule follow a "random error", and thus that the zero deviation (the mean) is the best expression of the true mean-the type.

Later, we will find certain cases where the distribution of variation is not "binomial" and where one cannot say that the mean should be used for exact investigations. According to the distribution, and therefore the variation curve, a pronounced obliqueness (asymmetry) or even two or more peaks may appear. In such cases one could choose several types. In this preliminary sketch, however, we will only consider cases where the variations are distributed in a tolerably and consistently binomial fashion. As we only ever take one character into consideration at a time, we will take that measure or intensity which is a "typical" mean of the given character in sequences of experiments as the "simple type" ${ }^{6}$ The types indicated just now for bean length or ray number are then two examples of simple types, because in each of these two examples only one type is worth mentioning.

It is such simple types that we consider here. Therefore, if we speak of types in at this point, we will mean types that appear as quantitative measures from series of experiments.

\footnotetext{
${ }^{6}$ We will mention compounded types ("complex types"), which is to say aggregate types, in the 19th lecture.
} 


\section{WILL THE TYPE BE SHIFTED BY SELECTION?}

The question then becomes this: will the selection of plus- and minus-varying offspring yield a shift in their type, in either a positive or negative direction? Galton's efforts appear to answer this in the affirmative. In England especially, it is a very common perception that Galton's investigations have provided solid evidence that selection can lead to the development of new races when followed for some generations, which is to say that selection leads to a type that varies from the original.

It is well-known that Darwin considered small individual variations to be meaningful for evolution by natural selection (even the swings of fluctuating variation 7 ). This is particularly true of the speculative biologists, either "Darwinists" or the so-called "ultraDarwinists", such as Wallace and Weismann. It must be noted here that Darwin also endorsed the importance of sudden jerky variations (his "single variations", or mutations), and also the possibility of a direct influence of the environment.

Galton's works and the subsequent studies of Pearson (among others) describe the foundation on which the present study of selection rests, namely selection on fluctuating variation. This teaching is also called the "continuous" evolution, in that it considers jerky ("discontinuous") variation as unsuitable for the appearance of new types. Indeed, the adherents of "continuous" evolution concept mostly doubt the existence of a difference between fluctuations and jerky variations. We cannot settle this question immediately and exhaustively, but only briefly say that such a difference can only emerge through the circumstances of heredity.

In any case, one must however give Pearson his due when he says the following: "If Darwinism is a true theory of evolution, that means

\footnotetext{
${ }^{7}$ MOP note: an old term signifying supposedly non-heritable variations, see De Vries' Mutation Theory for a discussion.
}

we should describe evolution by natural selection together with heredity, as that law, that clear and precise rule expressed in the typical character of the offspring as the function of the character of the ancestor, it becomes likewise a cornerstone of biology and the foundation on which heredity becomes an exact discipline. ${ }^{8}$

We must therefore test these causes, such that we are completely clear whether in reality a type can be shifted through biased selection of the plus- (or minus-) variations. We will now consider Galton's human data more closely. Were all offspring of parents exceeding 70 inches in body length collected, we would obtain the following succession of variations of the grown children (Table 1), where obviously the data for daughters are corrected following Galton. The material is divided into classes in 2-inch increments 9

The average of all 134 variations is $M=$ $70.15^{\prime \prime}, \sigma= \pm 2.29^{\prime \prime}$ and from that $m=$ $\pm 0.20^{\prime} 10$

The "theoretical" numbers are computed from $M$ and $\sigma$ as customary (see p. 67 in the original). With some good will one must acknowledge that the actual and theoretical numbers agree well enough. The obliquity of the distribution is only apparent; it is found also in the theoretical numbers. We will consider such distributions later, in which case the conclusion is obviously distorted somewhat. However, one cannot deny that this group of offspring has a "type" in the sense of Quetelet.

We find the same with the offspring of the minus-variants under 67", which appear as in Table 2.

From these 181 variations we have $M=$ $66.57^{\prime \prime}, \sigma= \pm 2.31^{\prime \prime}$, and $m= \pm 0.17$. Here also we can speak of a "type".

\footnotetext{
${ }^{8}$ MOP note: This is my translation back to English of Johannsen's rendering in German of Pearson's uncited text. I am not sure what the ultimate source of this quotation is, and I'm afraid that it is a bit mangled from the (presumably English) original.

${ }^{9}$ These classes are not precisely given by Galton.

${ }^{10} \mathrm{MOP}$ note: This last is the standard error of the mean, e.g. $2.29 / \sqrt{139}=0.198$.
} 
Table 1: Body length of the offspring of parents with average height above 70".

\begin{tabular}{llllllll} 
Class boundaries & $60.7^{\prime \prime}, 62.7^{\prime \prime}$ & $62.7^{\prime \prime}, 64.7^{\prime \prime}$ & $64.7^{\prime \prime}, 66.7^{\prime \prime}$ & $66.7^{\prime \prime}, 68.7^{\prime \prime}$ & $68.7^{\prime \prime}, 70.7^{\prime \prime}$ & $70.7^{\prime \prime}, 72.7^{\prime \prime}$ & $72.7^{\prime \prime}, 74.2^{\prime \prime}$ \\
\hline Count & 1 & 6 & 23 & 50 & 34 & 19 \\
Theoretical count & 0 & 1 & 8 & 26 & 45 & 36 & 18
\end{tabular}

Table 2: Body length of the offspring of parents with average height below $67^{\prime \prime}$.

\begin{tabular}{llllllll} 
Class boundaries & $59.7^{\prime \prime}, 61.7$ & $61.7^{\prime \prime}, 63.7^{\prime \prime}$ & $63.7^{\prime \prime}, 65.7^{\prime \prime}$ & $65.7^{\prime \prime}, 67.7^{\prime \prime}$ & $67.7^{\prime \prime}, 69.7^{\prime \prime}$ & $69.7^{\prime \prime}, 71.7^{\prime \prime}$ & $71.7^{\prime \prime}, 73.7^{\prime \prime}$ \\
\hline Count & 3 & 29 & 70 & 45 & 11 & 1 \\
Theoretical count & 3 & 16 & 45 & 61 & 41 & 13 & 2
\end{tabular}

Table 3: Body length of the offspring of parents with average height $67^{\prime \prime}-70^{\prime \prime}$.

\begin{tabular}{lllllllll} 
Class boundaries & $59.7^{\prime \prime}, 61.7$ & $61.7^{\prime \prime}, 63.7^{\prime \prime}$ & $63.7^{\prime \prime}, 65.7^{\prime \prime}$ & $65.7^{\prime \prime}, 67.7^{\prime \prime}$ & $67.7^{\prime \prime}, 69.7^{\prime \prime}$ & $69.7^{\prime \prime}, 71.7^{\prime \prime}$ & $71.7^{\prime \prime}, 73.7^{\prime \prime}$ & $73.7^{\prime \prime}, 75.7^{\prime \prime}$ \\
\hline Count & 1 & 16 & 76 & 174 & 201 & 114 & 26 & 5 \\
Theoretical count & 2 & 17 & 77 & 173 & 196 & 112 & 32 & 5
\end{tabular}

Table 4: Body length of the offspring of all parents in Galton's data.

\begin{tabular}{|c|c|c|c|c|c|c|c|c|}
\hline Class boundaries & $59.7^{\prime \prime}, 61.7$ & $61.7^{\prime \prime}, 63.7^{\prime \prime}$ & $63.7^{\prime \prime}, 65.7^{\prime \prime}$ & $65.7^{\prime \prime}, 67.7^{\prime \prime}$ & $67.7^{\prime \prime}, 69.7^{\prime \prime}$ & $69.7^{\prime \prime}, 71.7^{\prime \prime}$ & 71.7", 73.7" & $73.7^{\prime \prime}, 75.7^{\prime \prime}$ \\
\hline I. Plus-variants & 1 & 1 & 2 & 11 & 41 & 38 & 31 & 9 \\
\hline II. Average parents & 1 & 16 & 76 & 174 & 201 & 114 & 26 & 5 \\
\hline III. Minus-variants & 3 & 22 & 29 & 70 & 45 & 11 & 1 & 0 \\
\hline All offspring & 5 & 39 & 107 & 255 & 287 & 163 & 58 & 14 \\
\hline Theoretical count & 6 & 35 & 123 & 244 & 272 & 172 & 61 & 13 \\
\hline
\end{tabular}

Table 5: Offspring of hybrids of short and long beans.

\begin{tabular}{|c|c|c|c|c|c|c|c|c|c|c|}
\hline Class limits (mm) & 9,10 & 10,11 & 11,12 & 12,13 & 13,14 & 14,15 & 15,16 & 16,17 & 17,18 & 18,19 \\
\hline Individual counts & 2 & 20 & 136 & 540 & 1068 & 1125 & 636 & 180 & 18 & 0 \\
\hline Theoretical counts & 2 & 22 & 149 & 544 & 1060 & 1108 & 621 & 187 & 20 & 2 \\
\hline
\end{tabular}


We can now consider the parents of average size, to exhaust Galton's data. Out of these 613 individuals (Table 3), $M=68.06^{\prime \prime}, \sigma= \pm 2.31^{\prime \prime}$, and $m=0.094^{\prime \prime}$. The agreement with the theoretical distribution is very good. Here, the "type" agrees completely with the original population. A selection of "average-sized" parents should bring forth no alteration of type.

Galton's material consequently shows us that, through selection, the plus-variants above $70^{\prime \prime}$, the minus-variants below $67^{\prime \prime}$, and the average individuals between 67-70" yield three groups with distinct body-length types (results in English inches):

- Plus-variants: $70.15 \pm 0.20$

- Average parents: $68.06 \pm 0.09$

- Minus-variants: $66.57 \pm 0.17$

The differences between these three estimates are so large relative to their mean errors that there are very clear differences (after the results of page 86 this is easy to prove), such as those observed after selection.

What Galton obtained for body length will also hold for other characters.

We might therefore let ourselves be guided by the mathematicians and other mathematically qualified workers (like Quetelet, Galton, Pearson, and others), who have had such influence on the conception of the terms "type" and "typical" in heredity. If so, then we would have to say that selection truly shifts the type in the direction of selection.

If we instead cast a critical eye on Galton's results, we might note that a distribution agreeing well with the binomial distribution may contain more than a single type ${ }^{11}$ Specifically, if we more closely inspect the offspring data as a whole, we find that the individuals of the three types group very neatly as a single collective type. If we combine the three named group ${ }^{12}$, we obtain the following summary

\footnotetext{
${ }^{11}$ Except for a "pollution" of the series of variations through the presence of a single or only a few individuals of a different type. Such a pollution would alter the type of Quetelet only slightly in these data.

${ }^{12}$ The division is here somewhat different from that carried out earlier in this chapter, to allow agreement between
}

(Table $4^{13}$ ).

The mean of all 928 variations is $M=$ $68.086^{\prime \prime}, \sigma= \pm 2.54^{\prime \prime}, m=0.085^{\prime \prime}$. The theoretical counts, computed in the normal fashion, agree even better with the observations than for the individual groups I and II. This is likely due merely to the greater number of individuals.

The collected offspring give the impression of belonging to a single type, although we know that they may belong to at least three types! The only difference between the individual groups I-III and the total data is that the standard deviation of the last group is relatively somewhat larger, in that the coefficient of variance of the individual groups is 3.3-3.4, whereas in the total data it is 3.7. (Calculation conducted according to the method of page 48).

Therefore, there is greater variability in the total data than in the individual groups ${ }^{14}$ However, any sign that this data contains more types is not evident.

Thus, we can already state with confidence that we cannot determine whether there is one or more types within a set of variations whose distribution corresponds to the binomial distribution. Also, we cannot know whether the individual groups I-III are themselves mixes!

The "type" in Quetelet's sense is therefore merely a statistical idea. Such a type is the the immediate observation of a center for an available population or stock, around which the plus- and minus-variants of the character in question are grouped. However, this is a purely descriptive phenomenon. From it we can say nothing about whether such a type is singular or multiple groups of different nature are masked therein. This question can only be

groups II and III. [MOP note: unclear what he means here; perhaps the different body length bins?]

${ }^{13}$ MOP note: Johannsen's table uses somewhat weird orthography, such that the format shown here is unable to actually display an additional two observations in the theoretical counts, one greater than 73.7" and one less than 59.7".

${ }^{14}$ This is absolutely not always the case. One or a few of the individual groups can be more variable than the relevant total material. A small coefficient of variance cannot be taken as a sign of the presence of a single type only. 
answered through further biological analysis and, as we will see, through testing of heritability.

Altogether it can be asserted that, for a set of variants that has no typical distribution in Quetelet's sense (i.e. where the variation curves show a large shift or more than one peak), the mean has no meaning as a "typical" value ${ }^{15}$ However, the converse may not be concluded either: the most beautiful "typical" distribution may not be taken for uniformity of an apparent type.

Thus, the very beautifully "typical"appearing distribution curves used as examples (figures on page 74 and 75) give absolutely no guarantee that the mean length of the measured beans, and similarly the mean ray number of the flounders, are typical expressions of characters of a uniform nature in those groups of plants or beans.

We can use a mixture of bean individuals as one example where different biological types have been proved to appear together, but where there remains a perfectly good binomial distribution. The beans in question were all offspring of a hybrid derived from crossing of long and short races. The lengths of these offspring are given in millimeters in Table 5.

The mean of all 3718 variants is $M=14.072$ $\mathrm{mm}, \sigma=1.232 \mathrm{~mm}$, and the error of the mean is $m=0.20 \mathrm{~mm}$.

The theoretical numbers, computed as usual from $M$ and $\sigma$, agree well with the findings overall. Therefore, this appears once more to be a uniform type in the sense of Quetelet. In reality, however, there are (relative to measured length) three biologically distinguishable types: the type of the short bean, the type of the long bean, and a type that may here be called the hybrid type. The variations of these three types are collected in Table 6, from which the means and their errors and standard deviations may be seen.

Many more examples could be given showing that mixed populations are in good agree-

\footnotetext{
${ }^{15}$ Examples of such sets of variations will be given in the chapters 12 through 14 .
}

ment with the binomial distribution 16

\section{Phenotypes, genes, And GENOTYPICAL DIFFERENCES}

Thus we realize that the "type" in Quetelet's sense is only a superficial representation of nature, which may be deceptive. We must determine through further investigation whether one type or several biologically different types are present. We may thus refer to the statistically-derived type as an "apparent type" or, rather more briefly and clearly, as a "phenotype" ${ }^{17}$ Such phenotypes are in themselves measurable realities, as characteristic observations: the centers of datasets, around which variations are grouped. For this phenotype idea, we must only remember that no farreaching conclusion may be drawn from such phenomena. Some given phenotype may express a biological entity, however it is in no way required to do so. The phenotypes found in nature by statistical analysis of variation are generally not biological entities!

If we now use the word "phenotype" in the more precise meaning given here, we can say the following: in Galton's investigations, phenotypes are shifted by selection. However, the substance and the importance of this fact are not yet elucidated.

Before we examine selection and the phenotype more deeply with this new understanding, we must still clarify some points. The difference between different species or genera, for example between dog and cat, rose and lily-or between cat and rose, dog and lily-is at least in the greater part due to corresponding differences in the sexual cells of each organism. It cannot be doubted that the sexual cells-the gametes, as one says to mean the egg and sperm cells both-contain "something" that influences or defines the character of the organ-

\footnotetext{
${ }^{16}$ MOP note: This phenomenological observation would be formalized by Fisher in his famous 1918 paper.

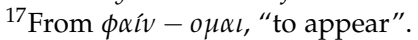


ism that they create by fertilization. The zygote (the product of the union of the two gametes in question) contains that same "something", now brought together through the union of the two gametes. This "something" in both the gametes and the zygote is commonly and ambiguously named "Anlagen' 18 which have substantive meaning for the character of the organism. Many other expressions have been proposed, most of these unfortunately being associated with specific hypotheses. Darwin's word "pangene" is the most common beside "Anlage".

The word "pangene" is rather poorly conceived, as it has an unnecessary second root,

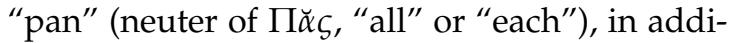
tion to "gene" (from $\gamma i-\gamma(\varepsilon) v-o \mu \alpha l$, "to become"). Only the meaning of this second root comes into play, merely expressing the simple notion that a character of a developing organism is or can be determined (or co-determined) through "something" in the gametes. No hypothesis about the quality of this "something" should be posed or supported thereby. Therefore it seems simplest to isolate this interesting last syllable "gene" on its own, to replace the word "Anlage". We will thus henceforth for "pangene" and "pangenes" instead say "gene" and "genes". The word "gene" is completely free of any hypothesis, but instead expresses the sure fact that each of the many characters of the organism are attributable to specific, separable (therefore independent) "conditions", "causes", or "Anlagen" received through the gametes; briefly, what we want to name by "gene" 19

The difference between rose and lily, between dog and cat, etc. is determined thus, namely that the gametes and zygotes in each case have different genes. (It should absolutely not be said that no similar or even identical genes may also be present; common characters

\footnotetext{
${ }^{18} \mathrm{MOP}$ note: I preserve the German word here. A common term of the time that is still used sometimes in English due to its generality. It can be translated as any one of the (plural) nouns "tendencies", "predispositions", "talents", "primordia", "complexes", or many others, depending on context.

${ }^{19} \mathrm{MOP}$ note: This paragraph contains, as far as I can tell, the original coining of the word "gene".
}

and tendencies are frequently found in quite different organisms.)

It will be seen that the short word "gene" provides many advantages, through the ease of combining it with other terms. And if we think of a character that is determined by a particular "gene" (or through a specific class of genes), we can quite easily say "the gene for the character", instead of the inconvenient phrase "the gene that determines the character" or similar expressions.

No satisfactory conception regarding the nature of the "gene" is currently established. This, however, has absolutely no influence on the virtues of heritability research; it suffices that it is certainly proved that such "genes" do exist. This proof, accomplished by Gregor Mendel (which we will deal with later ${ }^{20}$, is one of the most important achievements of precise research in experimental crossing. We find that the genes for very many characters are plainly separable, while others are not separable, or at least not neatly. This is reminiscent of the behavior of chemical bodies. That is however not to say (or at least not yet) that the genes are chemical entities-on this question, we can say nothing as of now.

Only this is certain: the various gametes contain specific, mutually separable "genes" for different characters. The gametes of, for example, Lychnis diurna contain genes for hairiness, genes for red color, genes for chlorophyll synthesis, etc. Each character based on a specific gene (or genes of a specific type) can also be described as a single character. The substance of such work is to test in each case which characters are uniform. Crossing experiments are a very important technique for studying such characters.

\footnotetext{
${ }^{20} \mathrm{MOP}$ note: he means in a later untranslated chapter.
} 
Table 6: Offspring of selected hybrids, ordered by biologically determined type.

\begin{tabular}{|c|c|c|c|c|c|c|c|c|c|c|c|}
\hline Class limits (mm) & 9,10 & 10,11 & 11,12 & 12,13 & 13,14 & 14,15 & 15,16 & 16,17 & 17,18 & Overall $M \pm m$ & $\sigma$ \\
\hline \multicolumn{12}{|l|}{ Individual counts } \\
\hline Short type & 1 & 15 & 98 & 277 & 229 & 50 & 1 & 0 & 0 & $12.800 \pm 0.035$ & 0.901 \\
\hline Hybrid type & 1 & 5 & 34 & 236 & 729 & 758 & 229 & 15 & 2 & $13.971 \pm 0.021$ & 0.954 \\
\hline Long type & 0 & 0 & 4 & 27 & 110 & 317 & 406 & 165 & 16 & $15.082 \pm 0.032$ & 1.031 \\
\hline In total & 2 & 20 & 136 & 540 & 1068 & 1125 & 636 & 180 & 18 & $14.072 \pm 0.020$ & 1.232 \\
\hline
\end{tabular}

Table 7: Averages and variances for length and breadth of four types of bean.

\begin{tabular}{llllll} 
Offspring series & Bean length mean $(M \pm m)$ & $\sigma$ & Bean breadth mean $(M \pm m)$ & $\sigma$ & Number of individuals \\
\hline BB & $11.206 \pm 0.008$ & 0.726 & $8.091 \pm 0.004$ & 0.400 & 8491 \\
E & $12.793 \pm 0.011$ & 0.747 & $9.379 \pm 0.007$ & 0.468 & 4949 \\
GG & $12.942 \pm 0.015$ & 0.813 & $8.152 \pm 0.007$ & 0.405 & 2937 \\
MM & $14.405 \pm 0.009$ & 0.900 & $7.976 \pm 0.004$ & 0.348 & 9440
\end{tabular}

Table 8: Degree of serration in two kinds of barley.

\begin{tabular}{lllllllllllll} 
\% serrated & $0 \%$ & $5 \%$ & $10 \%$ & $15 \%$ & $20 \%$ & $25 \%$ & $30 \%$ & $35 \%$ & $40 \%$ & $45 \%$ & $50 \%$ & Number of individuals \\
\hline Series A & 209 & 9 & 4 & 1 & 0 & 0 & 0 & 0 & 0 & 0 & 0 & 223 \\
Series B & 0 & 0 & 0 & 0 & 9 & 49 & 63 & 33 & 12 & 4 & 5 & 175
\end{tabular}


The clearest cases are those in which different characters can be treated as qualitative. Although such different characters sometimes appear linked (or continuous), they are the easiest to define into single characters. It was simple to determine that "redness" or "hairiness" of Lychnis diurna are single characters determined by different genes, and analysis in this case provided immediate insight with hardly any difficulty. It was also easily demonstrated that the different bean coat pigments (yellow, violet, bronze etc.) are determined by specific genes. Similarly, certain pea varieties have specific genes that produce green or yellow seeds, etc.; many of the morphological characters of plant and animal species are determined by specific genes or gene complexes. Also, purely negative characters like hornlessness in cattle or albinism (whiteness) in many flowers can occur due to the failure (or inefficacy) of genes or through the inhibitory action of genes.

All such characters are conspicuous and therefore easily accessible to analysis and immediately understandable to the observer. In these examples one deals with qualitatively different characters with no quantitative ambiguity; they are really cases of alternative characters.

However, in this lecture we must next deal with numerical variability: quantitative differences between variations measured on the same scale. Therefore alternative variations are irrelevant; rather we deal with grades or intensities of measurable quantities.

If we compare different species or races, we find not only morphological or other obviously qualitative differences, but also differences of degree related to dimension, color intensity, chemical content, and various other characters that are numerically specified. Between two similar races, only such a quantitative difference may be present or observable.

As one example, different bean races can be selected that only (or nearly only) differ in that their seeds are different in length and breadth. See Table 7 for the average values of length and breadth measurements for four purebred series of offspring of brown princess beans (Phaseolus vulgaris) grown together in 1903.

There are very obvious differences here. The standard errors $(m)$ of the means $(M)$ are relatively small due to the large quantities. We immediately see four different phenotypes, for example for length. What is the cause of this difference?

Let us select a further example. I compared serrations ${ }^{21}$ on the ears of two purebred lines ( $A$ and $D$ ) of distichous barley were grown together in the same field in the same year (1904; Table 8). For series A the mean is $M=$ $2.95 \pm 0.13 \%$ serrated with $\sigma=1.90 \%$, whereas for series $\mathrm{D}$ we find that $M=33.13 \pm 0.48 \%$ with $\sigma=6.32$. For series A serration is essentially absent, whereas series D is starkly serrated. Obviously, we have two different phenotypes here. And we can ask here, as before, what is the reason for this difference?

After further investigations it has been shown that, in each of these two examples, the distinction in phenotype corresponds to an actual difference of genes. Differences between these phenotypes are determined by (or contingent upon) the presence of different genes because the particular phenotypes are not only superficially distinct, but also distinct on more fundamental levels. Such differences are of a so-called "genotypical" nature, in that the types in question are different with respect to their genes ${ }^{22}$

However, as we proceed we will find that phenotypic and genotypical differences do not by any means need to be congruent. In each case in-depth analysis is needed to show whether genotypical differences are present or not; an inspection alone cannot decide this matter.

\footnotetext{
${ }^{21} \mathrm{By}$ 'serration' I mean the occasional appearance of some number of young fruiting nodes that do not develop to grains. The ripe ears then contain empty places, socalled "notches" or "gaps". The serration of a plant can be measured through the count in percentage of the notches out of the total number of "grain places" in the plant.

${ }^{22}$ MOP note: This is the first recorded usage of the term "genotypic"/"genotypical" or "genotype". See Johannsen 1911 American Naturalist $p .133$ for why he prefers the adjective: "We do not know a 'genotype', but we are able to demonstrate "genotypical' differences." I kept the "-al" suffix as this appears to be preferred by Johannsen and early authors in English.
} 


\section{Homozygotes AND HETEROZYGOTES}

Before we proceed further, we must examine some expressions that are in general usage in the heritability field. When fertilization occurs, two cases are possible with relation to the genes: 1) the two conjugating cells have genes of a similar nature, or 2) they have genes of a different nature. In the first case, a "homogeneous" zygote will develop, whereas in the second a "heterogeneous" zygote will result. Because obviously these words are used for many other meanings, it would be rather impractical to use these words here for a special purpose. Therefore, one can instead take Bateson's suggested terms "homozygote" and "heterozygote" if one wishes to state that a zygote of united gametes (egg or sperm cells) are alike or unlike with respect to their genes.

A homozygous state therefore arises from the union of gametes with like genes, and may therefore be called "pure" or "racially pure" ${ }^{23}$ A heterozygous state is produced from gametes that are not identical with regard to genes. Such a state has a hybrid nature. And if the gametes in question are different in one or two or more points with regard to the genes, the state that arises through fertilization will be heterozygous in one, two, or more respects. We discuss this more in the 22nd lecture of this book.

\footnotetext{
${ }^{23} \mathrm{MOP}$ note: These were unfortunately the ways that people applied categories in that time. Obviously, we now know that there is little relationship between human sociological "race" and genetics, beyond a famous but rather meaningless neutral signal of population structure. After some consideration, I chose to translate this directly; this terminology is far outdated and offensive, but it is also the way that people spoke and thought once, and to some extent still speak and think today.
} 